\title{
Tangence
}

\section{La liberté d'un homme selon Arthur Villeneuve}

\section{Michaël La Chance}

Numéro 52, septembre 1996

Tours et détours du romanesque : Minuit aujourd'hui

URI : https://id.erudit.org/iderudit/025920ar

DOI : https://doi.org/10.7202/025920ar

Aller au sommaire du numéro

Éditeur(s)

Tangence

ISSN

0226-9554 (imprimé)

1710-0305 (numérique)

Découvrir la revue

Citer cet article

La Chance, M. (1996). La liberté d'un homme selon Arthur Villeneuve.

Tangence, (52), 105-112. https://doi.org/10.7202/025920ar d'utilisation que vous pouvez consulter en ligne.

https://apropos.erudit.org/fr/usagers/politique-dutilisation/ 


\section{DUCWMPNT}

\section{La liberté d'un homme selon Arthur Villeneuve}

\section{Michaël La Chance}

Ces réflexions sont issues de conversations avec Artbur Villeneuve à l'automne 1988. Nous devions réaliser un livre d'eaux-fortes sur cuivre, un bestiaire inspiré des planches de l'Histoire naturelle de Buffon. Léo Rosshandler avait accepté de rédiger une présentation. De ce livre, qui n'a pas vu le jour en raison du décès d'Artbur Villeneuve en 1990, reste ce texte auquel je donne le titre que le peintre avait lui-même suggéré.

La plupart semblent croire qu'ils sont libres dans la mesure où il leur est permis de vivre selon leur penchants [...]

[c'est] la crainte d'être punis par d'horribles supplices après la mort, qui les pousse à vivre selon les prescriptions de la loi divine [...]

s'ils croyaient au contraire que les esprits périssent avec le corps [...] ils voudraient tout gouverner selon leurs penchants et obéir à la fortune plutôt qu'à eux-mêmes. Ce qui me paraît pas moins absurde que si un homme, parce qu'il ne croit pas pouvoir nourrir éternellement son corps de bons 
106

aliments, préférait se saturer de poisons mortels; ou bien parce qu'il voit que l'esprit n'est pas éternel ou immortel, préfère être dément.

Baruch de Spinoza ${ }^{1}$

\section{Importance}

Lorsqu'on considère l'homme, il semble que rien ne l'empêche de jouir de la vie. Pourtant c'est lui-même qui s'y refuse. Nous devons sans relâche interroger chez l'homme cette tendance inavouée pour tout ce qui lui est contraire.

Comment paraître naiif. Non pas refuser le monde des mots et des images qui est partagé de tous, mais feindre ne pas les comprendre pour se rendre compte qu'effectivement on ne les comprend pas. Ce que nous appelons le temps, l'espace, le monde ce ne sont que des images.

Par défaut de comprendre le monde des images dans lequel nous appréhendons la réalité, le naïf se soustrait à une tyrannie de la description. Ce qui, cependant, ne le met pas à l'abri des descriptions d'autrui et ne lui donne pas la force de leur résister, quand elles semblent avoir prise sur lui.

Arthur Villeneuve ne prétend pas avoir trouvé une autre façon de voir le monde mais il nous le laisse envisager dans des images qui ne disent pas tout, qui montrent la trop grande simplicité des représentations et des explications. Sa peinture met en évidence le caractère simpliste de notre façon de représenter: nous sommes des naïfs qui nous ignorons. Pour sa part il ne sera pas assez naif de croire que l'on peut ouvrir dans la façade des descriptions partagées de tous une fenêtre sur le monde vu autrement.

Certes, lorsque nous regardons une peinture d'Arthur Villeneuve il nous paraît naif. Est-ce dire que nous le sommes moins? Nous croyons ne pas être naifs, mais alors que sommes nous? Nous ne sommes tout au plus que des hommes abstraits. L'homme qui n'est pas libre est celui qui laisse le temps passer

1 Baruch de Spinoza, L'Éthique, ve partie, - De la liberté humaine •, scolie prop. XI,I; O.C., trad. R. Caillois, Bibliothèque de la Pléiade, p. 595. 
sans profiter de ce bien, la vie: il se contente de l'idée qu'à chaque instant tout est possible. Il a l'idée qu'il peut tout entreprendre sans rien commencer. Il parvient à jouir de ce qu'il ne fait pas, qu'il ne possédera pas ou n'accomplira pas, mais qu'il croit pouvoir accomplir un jour — tant que cela ne lui paraît pas impossible et qu'il n'en fait pas son deuil. Il fait sa richesse de tout ce qui est abstraitement possible, il est riche de tout ce qui ne lui est pas retiré, même s'il n'en n'a que l'idée.

Pour certains les loteries recréent à chaque jour la richesse. Pour Arthur Villeneuve la peinture est un pari pour l'improbable par lequel il gagnait sa liberté.

Alors il faut dire que sa peinture est l'exercice de la liberté qu'il trouve - elle est un moment d'absurdité réelle qu'il peut se donner.

Sans chercher à se donner l'importance à laquelle tout le monde s'emploie.

\section{Méfiance}

Devant les autres nous avons peine à dissiper le sentiment qu'ils sont improbables, que nous sommes tous improbables, alors la panique est la situation limite. Nous n'avons rien à craindre sinon l'effondrement - qui ne sera pas nécessairement remarqué. Arrive un moment où nous ne saurons plus reprendre la conversation, où nous ne saurons même pas avouer notre extrême fatigue. Cette prostration nous permettra de tenir le coup encore un temps, plié sur un sol qui bascule, pour ne pas sombrer dans une. crise insondable.

Pour ne pas sombrer, il faut n'avoir de cesse d'interroger la vie humaine, de s'étonner du destin de chacun.

C'est ainsi que l'effort de paraître informé, à l'aise, à sa place - nous laisse épuisé. Quand vient le temps de s'enfouir de nouveau en soi-même on n'y retrouve plus que peur et confusion. Trop vidé par le monde de ce qu'il faut dire, par l'effort terrible que demande chaque banalité - on ne sait plus amorcer une phrase en soi-même, pour soi-même. Le miroir de l'âme ne connaît plus son lustre profond.

Faillir à sa liberté c'est s'habiller d'une description de soimême, c'est emprunter des modes de satisfaction de soi. La 
108

société nous fait jouer un rôle et nous reproche en même temps de ne pas être authentique. Dans un monde qui va comme un vent froid et revient comme un écho lugubre, l'homme est toujours déporté hors de lui-même. La liberté triomphe lorsque chaque rencontre et chaque situation ne nous creusent pas d'un doute: - quelle a été ma conduite?

La liberté d'un homme c'est alors la capacité d'ouvrir sa vie présente, parce qu'elle est bien là. Une vie aux bras multiples, qui connaît tous les refuges du regard. Dans cette vie nous pouvons accueillir tant de choses, en celle-ci les choses nous trouvent déjà recueillis devant elles.

Sinon on croit que la vie va commencer plus tard, qu'elle est beaucoup plus compliquée, qu'il faut être naif pour croire que les choses sont simples. Terrorisés par l'énormité de cette vie qui va commencer, nous sommes ébranlés, fermés sur nous-mêmes, sans que le talent et l'apprentissage puissent rien déposer.

\section{Impécuniosité}

Arthur Villeneuve vivait comme un dilemme personnel le fait d'être placé entre deux chaises, celle du barbier et celle de l'artiste en son atelier. Le client peut commander sa coupe au coiffeur mais l'artiste Villeneuve réclame une liberté qui n'est pas cautionnée par une connaissance de l'art. La société dit de l'artiste: il peut faire ce qu'il veut mais il doit savoir ce qu'il fait. Villeneuve cautionnait sa liberté créatrice autrement. Chaque métier préconise une façon de se créer une réputation. La réputation du barbier devient la conséquence naturelle de sa fierté d'artisan. Par contre Arthur Villeneuve ne pouvait certifier sa réputation d'artiste par l'éducation, le privilège de classe, l'appartenance à une coterie culturelle. Alors il lui a fallu se donner comme fondement son propre respect de soi-même, protéger ce respect du doute qui constamment l'assaille, l'alimenter de toutes les formes d'attention qui pouvait le concemer. Telle est la véritable maison de Villeneuve.

Il y a tout ce que je garde chez moi pour me protéger du besoin. Il y a aussi tout le reste que j'accumule comme des trophées de l'aisance, ou encore par peur de manquer un jour. Pourtant, toutes ces choses par lesquelles le besoin fait sentir sa morsure m'encombrent. C'est une liberté que de pouvoir posséder peu, de posséder à l'abri des regards. 


\section{Invalidité}

Le secret des grandes promenades, à pied, à bicyclette: se donner un corps plus vaste, avec une inertie bienfaisante, où les secousses des nerfs vont s'amortir sous l'induration des chairs, où le tourment de l'âme prend forme. Lorsque le corps n'est pas secrètement tordu par les souffrances, alors il peut être un réservoir, cette machine qu'il suffit de laisser aller vers ses exploits.

La souffrance s'accumule dans les générations, détermine la forme de l'être. Et les êtres particuliers souffrent à leur insu de cette torsion, bientôt apparente par la souffrance qu'ils infligent aux autres. La souffrance s'installe d'abord comme une infime insensibilisation à la vie qui s'ajoute et se diffuse dans la vie de chacun. L'hébétude du corps donne leur consistance aux mots, l'extinction des sens donne ses illuminations aux images - et c'est ainsi que les mots et les images deviennent le raccourci le plus fulgurant pour devenir notre souffrance à chacun de nous, comme si nous n'étions que l'idée de ce que nous sommes, lorsque notre propos jour après jour profile un propos invisible, le propos invalide que nous sommes et que les autres sont également.

\section{Impasse}

Lorsqu'il n'avait aucune possibilité d'évasion, Arthur Villeneuve allait dans son sous-sol fabriquer des bateaux, des bâtiments miniatures - jusqu'au jour où il découvre qu'il peut les peindre. Chacun sa caverne et ses reflets caverneux. On ne voit pas autre chose que cela. Pour certains les signes traversent la pierre, certains découvrent Lascaux chez eux.

Approuver que l'on est, recevoir l'existence comme chance - cela nous vient facilement lorsque nous le pouvons sans démentir une culture qui semble dire qu'elle est le lieu le plus propice à l'épanouissement de l'humain. Il est plus facile d'approuver que l'on est lorsqu'il semble qu'en tous lieux on nous attend. Alors qu'adviennent ceux dont l'intelligence, la sensibilité, ne s'accordent pas à leur époque?

Comment l'accord avec sa propre existence est-il possible chez ceux qui ont dû tout sacrifier pour se donner une sécurité, pour ne pas apparaître inférieur? Il y a une gratuité de croire que l'épanouissement d'un être humain ajoute quelque chose dans l'univers 
et peut agir sur le destin de ceux qui suivront. Il y a une gratuité d'imaginer que tout bien-être répond à un appel, lorsque le sentiment de liberté en moi serait l'éclosion d'une partie du monde.

Est-ce que toute liberté s'accompagne d'un sentiment d'unité avec la création? Il y a cette naïveté dans le bonheur, la naïveté de croire qu'il suffit d'aborder de front la masse de son existence pour trouver le chemin de la vie et vivre profusément. Qui veut perdre cette naïveté?

Arthur Villeneuve refuse de parler de naïveté à propos de sa peinture, qu'il préfère caractériser de "lyrique". Mais il aime parler de la folie de ceux qui croient savoir, il aime parler de la liberté d'appartenir à tout ce qui est, d'obéir à une loi à l'œuvre dans la pleine étendue du monde, quand à chaque instant le monde nous en impose dans la magnificence et la grandeur.

La liberté c'est la possibilité, dans l'insistance d'un labeur, de se donner des capacités et de sentir, par cette contrainte que nous avons su nous imposer, que nous redevenons des vertébrés. En effet, lorsque nul n'a de mots pour qualifier ce que l'on est et ce que l'on fait, il faut du courage pour ne pas faire comme tous les hommes qui préfèrent *obéir à la fortune plutôt qu'à euxmêmes ", selon Spinoza.

\section{Indépendance}

Se tenir à distance de la culture, maintenir une méfiance viscérale envers les arts. Les œuvres exposées au public ne seraient que des relais naîfs dans une manipulation du public, où l'État projette une image de liberté et de modernité, où l'ordre culturel géré par l'institution et ses décideurs trouve sa légitimation avant même de légitimer les ouvres. L'artiste naif est le moins naif envers l'art? À quoi ressemblerait un scientifique naif? Un philosophe naif? Trouver douteux que, dans cette culture, quelque chose puisse à la fois avoir du sens et paraître clair.

Il y a un système des rapports humains qui passe à travers la gorge de la plupart, dans lequel pourtant quelques-uns prospèrent. Qui saurait se considérer artiste et tout à la fois accepter que l'art est défini par ceux qui en font avec succès? Comment peut-on se croire artiste quand il n'y a personne pour vous dire de continuer? Peut-on se croire humain lorsqu'il n'y a plus personne et que l'espoir est nourri par des calculs? 
Arthur Villeneuve, par la peinture qu'il fait, rappelle qu'il n'y a personne et que l'espoir doit être autrement altéré. En effet, l'artiste par le type d'art qu'il fait, et donc par le-succès ou la négligence même qu'il rencontre, n'offre-t-il pas une image de son existence? En acceptant le succès comme artiste, je prends le risque de réduire ma vie personnelle à une image de réussite.

Et c'est là qu'il refuse d'être naïf. Certains disent:

- Trouver le succès en art c'est être assuré d'une existence pleinement vécue, comme tout succès en offre la garantie.

Il faut répondre:

- Peut-être que le succès en art devrait signifier tout autre chose. Votre succès impose à tous les autres de se départager entre le ratage et la réussite, il impose un profil du succès auquel ils devront se soumettre, persuadés que la satisfaction de soi correspond à un véritable épanouissement personnel. Mais la satisfaction de soi ne provient-èlle pas du fait que l'on aime le rôle que l'on joue, quand c'est un rôle valorisé par la société, sans avoir conscience que l'on ne joue ce rôle qu'au détriment d'une réalité de l'être humain?

Est-ce que la réalisation de soi comme être humain permet de se développer comme artiste? Inversement, la société établit que la création chez l'artiste entraîne nécessairement un épanouissement chez l'individu. Il s'agit tout simplement pour cette société d'affirmer que la création est une activité auto-gratifiante, et d'entretenir ainsi un vivier de producteurs d'ouvres qui peuvent s'estimer heureux tout simplement de pouvoir en faire, qui ne sont pas payés au-delà et qui - au plus souvent- doivent continuellement payer de leur poche pour continuer à faire de l'art. Ces artistes, limités par leur attachement à l'"oeuvre", ne voient pas que l'art officiel est tout entier occupé à produire des scénographies monumentales à son public, et puise par moments dans le vivier des artistes de seconde catégorie pour y découvrir de nouveaux scénographes.

De façon tout à fait involontaire, Villeneuve a produit la scénographie monumentale qui assure aujourd'hui son succès muséal. Est-ce un hasard si sa maison peinte est devenue l'enjeu de la survivance de son œuvre au niveau de la culture officielle?

Il y a ainsi une certaine naïveté de croire que le choix se situe entre faire de l'art selon soi-même ou plutôt de singer la 
112

création de ceux qui ont du succès, soit encore de se mettre à la remorque de ceux qui définissent pour l'heure ce qu'est l'art. Les critères de succès ne sont pas tant qualitatifs (l'authenticité et l'originalité, être le premier dans une façon de faire de l'art, etc.) mais quantitatifs (l'échelle à laquelle l'œuvre peut être rendue visible, la portée des événements qui peuvent s'y greffer, etc.). C'est la raison de l'effort considérable que l'on déploie dans le milieu pour bien prendre la mesure de votre succès aussitôt que vous présentez les dispositions requises, dans une société qui évalue sa réussite globale à la mesure du succès qu'elle peut offrir à quelques individus.

Il y a une façon de faire de l'art qui se détoume résolument des avenues du succès, des modes, des tendances officielles. Car tout succès est dû à une capacité de prospérer sur un état de fait, de prendre avantage d'une perversion incontournable des rapports humains. L'insuccès de quelques-uns tient davantage de l'obliquité de leur époque et non de leur incapacité de s'élever. On ne saurait prendre avantage d'un état de chose lorsque la stupeur que quelque chose existe nous tient à l'écart de tout. En ce sens là, Arthur Villeneuve ne réussira jamais. C'est sa liberté. 